\title{
Internal Consistency and Confirmatory Factor Analysis of Smartphone Addiction Inventory (SPAI)
}

\author{
Saeed Imani ${ }^{1}$, Jaber Alizadeh Goradel ${ }^{1}$, Sadegh Mousavi ${ }^{1}$ and Alireza Noroozi ${ }^{2}{ }^{2,3,{ }^{*}}$ \\ ${ }^{1}$ Department of Clinical and Health Psychology, Shahid Beheshti University, Tehran, Iran \\ ${ }^{2}$ Iranian National Center for Addiction Studies, Tehran University of Medical Sciences, Tehran, Iran \\ ${ }^{3}$ Neuroscience and Addiction Studies Department, School of Advanced Technologies in Medicine, Tehran University of Medical Sciences, Tehran, Iran \\ "Corresponding author: Iranian National Center for Addiction Studies, Tehran University of Medical Sciences, No. 486, South Kargar St., Qazvin Sq., Tehran, Iran. Tel: \\ +98-2155421144, Fax:+98-2155412232, Email: a_r_noroozi@yahoo.com
}

Received 2017 June 21; Revised 2019 March 04; Accepted 2019 May 21.

\begin{abstract}
Background: Parallel with the rapid growth of smartphone users among the youth, its problematic use has received attention due to adverse outcomes. The psychometric properties of smartphone addiction inventory (SPAI) have been studied among Italian and Taiwanese university students.

Objectives: The aim of this study was to investigate the psychometric properties of the SPAI among Iranian university students. Methods: We recruited 402 undergraduate university students in the study from February to March 2017. The SPAI and young's Internet addiction test (IAT) were completed for them. The AMOS statistical software (version 21) was used to test the five-factor model proposed in the original SPAI. The results of the goodness-of-fit index showed an ambiguous solution. For more appropriate trimming of the SPAI factorial structure, an exploratory-confirmatory cross-validation strategy was followed.

Results: The SPAI showed excellent internal consistency (Cronbach's $\alpha=0.92$ ). Cronbach's $\alpha$ values of the five subscales varied from 0.53 to 0.84 . The results supported a five-factor solution consisting of sleep interference, craving, daily life inference, compulsivity, and time spent $($ chi-square $=1105.445 ; \mathrm{P}<0.000 ; \mathrm{CFI}=0.902 ; \mathrm{TLI}=0.91 ; \mathrm{RMSEA}=0.09$ ), which showed good convergent validity with the IAT scores. Moreover, the SPAI showed high factor correlations.

Conclusions: Given the increasing trend of smartphone users in the country, the Persian version of the SAPI could be a useful tool for further investigation of smartphone problematic use among Iranian university students.
\end{abstract}

Keywords: Smartphone, Addictive Behavior, Psychometric

\section{Background}

The trend of smartphone use in recent years shows a dramatic increase and it has become part of the everyday life of a large proportion of populations, particularly the youth (1). According to Iran's Ministry of Information and Communication Technology in 2015, 27.5 million people were smartphones users in the country (2). During the last decade, the concept of addiction has been widening to include not only substance use, but also behavioral addiction including gambling, Internet use, and games $(3,4)$. The American Psychiatric Association (APA) reclassified the gambling disorder as an addictive disorder and listed Internet gaming disorder in section III of DSM-5 as an addictive disorder requiring further research (5).

There are similarities between smartphone addiction and Internet addiction as technology addiction. However, there is a more complicated interaction between individ- uals and their smartphones because of the multi-purpose application of smartphones (6). Since smartphones provide a wide range of services, their maladaptive use might not be evident at the early stages. Although there are no internationally approved criteria for smartphone addiction, researchers conceptualize it as a maladaptive pattern of smartphone overuse that results in the loss of control over its use and paying less attention to other important areas of functioning (6-10). Al-Barashdi et al. reviewed the signs and symptoms of addiction to smartphones. They recognized a number of features in their work including overuse and excessive time spent, emotional tension when smartphones could not be used, obsession to smartphones or a sense of anxiety and loss when not having access to phones, positive anticipation, difficulty in managing the use, helplessness in controlling the urge and craving, and daily life disturbance (7). Despite the benefits of using 
smartphone technology, the problematic use or overuse of smartphones could be associated with negative effects on mental health and psychosocial functioning $(7,11-14)$.

Given that the widespread use of smartphones is a relatively recent phenomenon, the most bodies of research on this type of addiction have focused on instruments designed to evaluate problems with mobile phones, such as the problematic mobile phone use (15), the mobile addiction test (16), the Manolis/Roberts cell phone addiction scale (17), the mobile phone problem use scale (18), and the cellular phone addiction scale (19). These tools mostly conceptualize the problem as a type of behavioral addiction featured by problems with impulse control for cell phone activities such as using the short message service (SMS), multimedia message service (MMS), and some Internet services. In contrast, a smartphone provides a convergence of services such as a camera, MP3 player, GPS, web browsing, calling, sending e-mails, gaming, and social networking services (SNS). To our knowledge, to date, there are only three instruments to assess smartphone addiction: the smartphone addiction scale (20), the smartphone addiction measurement instrument (21), and the smartphone addiction inventory (SPAI) $(6,22)$. Previous studies have shown the reliability and validity of the SPAI among Taiwanese (6) and Italian (22) university students.

\section{Objectives}

Given the increasing trend of smartphone users among the youth and the lack of screening questionnaires for smartphone addiction in the Persian language, the purpose of this study was to measure the psychometric properties of the Persian version of the SPAI in a sample of Iranian university students.

\section{Materials and Methods}

\subsection{Participants and Sampling}

A psychometric design was used to conduct the study. The study population included the undergraduate students of Shahid Beheshti University (SMU) located in the North of Tehran who were studying in the academic year of 2016 - 2017. A convenience sampling method was used to recruit the participants from February to March 2017.

Among 425 university students who consented to participate in the study, we excluded 23 participants who did not answer the study assessments completely. The final sample ( $\mathrm{n}=402 ; 72.63 \%$ female) reported a mean age of 21.98 years (range $=18-33, S D=3.18$ ). The inclusion criteria were being at least 18-years-old, being a university student, and using a smartphone while the exclusion criterion was not giving informed consent.

Participation in the study was voluntary and the participants consented to take part in the study. Participants completed the study instruments anonymously in privacy. They were assured that they could leave the study at any time they want, that their presence in the study was completely anonymous, and that they would opt for deleting or not giving any information about which they felt concerned. An identification number was assigned to each of the participants in order to identify the information related to them.

No criteria have been defined in the literature for the adequate sample size for studies measuring the psychometric properties of questionnaires. It has been recommended using at least 50 participants to measure reliability. The adequate sample size to conduct a factor analysis is subject to debate. There are many rules of thumb for determining adequate sample sizes for factor analysis including the rule of 5 (at least five cases per each item in the questionnaire) and the rule of 10 (at least 10 cases per each item in the questionnaire) with a minimum number of 100 participants to ensure the stability of the variancecovariance matrix (23). Some authors have recommended a minimum of 300 participants and an item-to-participant ratio of at least 5 to 10 (24). The SPAI questionnaire has 24 items. Given that there might be missing data, we considered a sample size of 425 . Before the confirmatory factor analysis, we conducted Kaiser-Meyer-Olkin (KMO) to measure sampling adequacy. The KMO index of sampling adequacy was 0.92 , which indicated the sample size was quite adequate.

\subsection{Tools}

\subsubsection{The Internet Addiction Test (IAT)}

The Internet addiction test (IAT) is a 20-item selfadministrated questionnaire developed by Young (25) with good internal consistency and concurrent validity $(26,27)$. Each item is scored based on a five-point Likerttype scale ranging from 1 (never) to 5 (always). The questionnaire has been validated in the Persian language with Cronbach's alpha of 0.89 (28). This measure assesses how much Internet use is associated with the loss of control and how much it negatively affects personal, interpersonal, and social functioning of the individual. We used the IAT scores to test the convergent validity of the SPAI. 


\subsubsection{Smartphone Addiction Inventory (SPAI)}

The smartphone addiction inventory (SPAI) is a selfadministrated questionnaire with items scored based on a four-point Likert scale from 1 (strongly disagree) to 4 (strongly agree). The questionnaire was originally developed to measure smartphone addiction based on Internet addiction features among the Taiwanese youth (6). The Italian version of the SPAI with 24 items showed excellent internal consistency and good convergent validity with IAT (22).

In order to collect the data regarding the gender, age, and academic majors of the participants, a specific questionnaire was developed and used by the researchers to assess the sociodemographic information of the participants.

The study protocol and the questionnaires were approved by the Ethics Committee of Shahid Beheshti University (No. 1395.781).

\subsection{Data Analysis}

We used SPSS-22 for descriptive analysis and AMOS statistical software (version 21) for confirmatory factor analysis of the data.

\section{Results}

We obtained written permission from Pavia et al. (22) for the translation of the SAPI. Then, two professionals who were fluent in both Persian and English languages translated the SPAI from the original English text into Persian. They compared their translations and discussed alternative words that best conveyed the concepts of the test. A third bi-lingual expert conducted a back-translation. There were minor conceptual and syntactical inconsistencies between the original English text and the back-translated version. After discussing and making adjustments, the final Persian version of the SPAI was prepared.

\subsection{Confirmatory Factor Analysis}

We used a five-factor model identified in the Italian version of the SAPI including time spent, compulsivity, daily life interference, craving, and sleep interference for confirmatory factor analysis. The goodness-of-fit index indicated a good fit of the model to the data (chi-square $=1105.445 ; \mathrm{P}$ $<0.000$; CFI = 0.902; TLI = 0.92; RMSEA = 0.09) using AMOS statistical software (version 21) and there was a deviation of the data from the model (Figure 1).

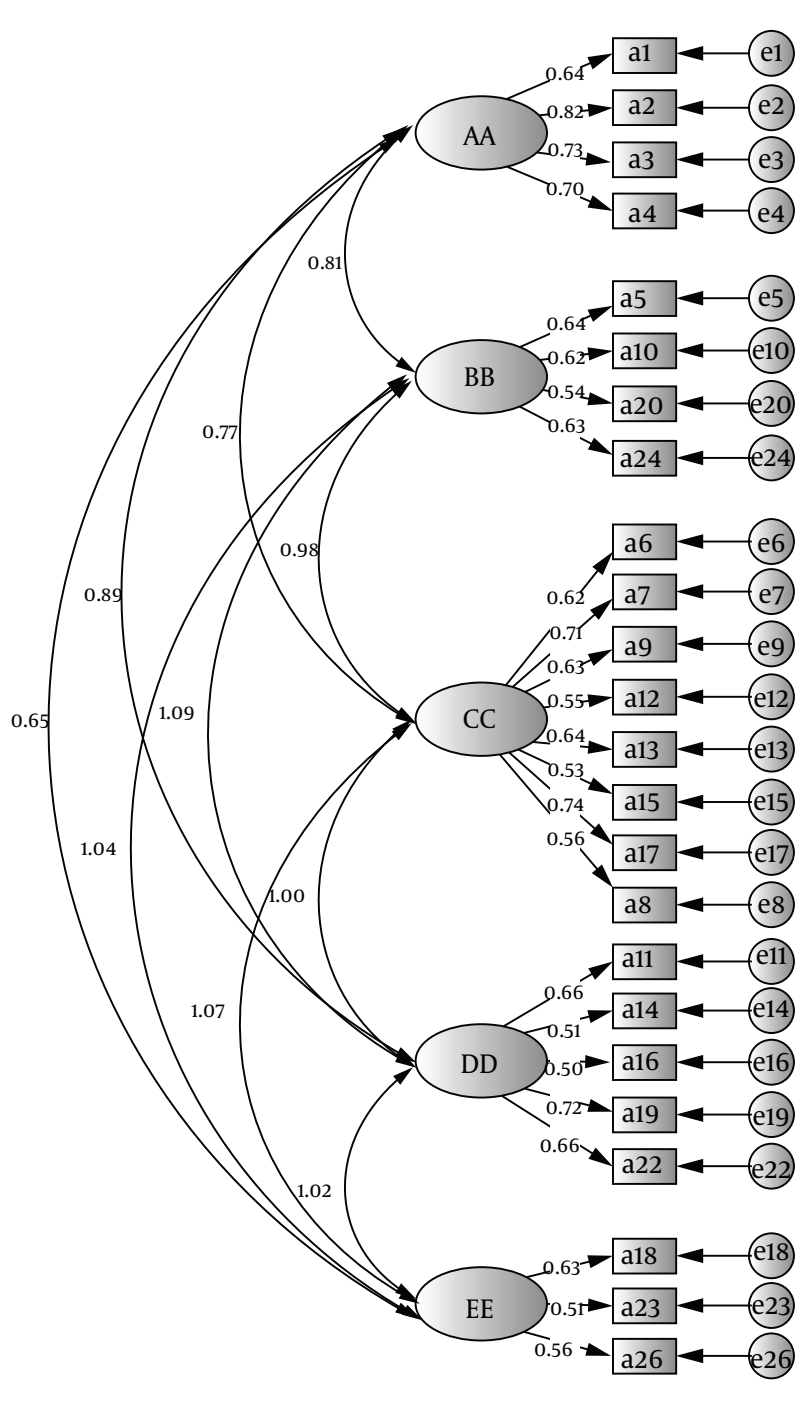

Figure 1. Confirmatory factor analysis

"Time Spent" (items 1 to 4 ) comprised four items that assessed the individuals' levels of difficulty in stopping using smartphones (e.g., "Q2: I feel uneasy once I stop using the Smartphone for a certain period of time") and dedicating more time and resources for using smartphones (e.g., "Q1: I was told more than once that I spend too much time on the smartphone"). "Compulsivity" (items 5, 10, 20, and 23) assessed the level of uneasiness and emotional suffering (e.g., nervousness, touchiness, and tension) felt by not using smartphones (e.g., "Q10: I feel distressed or down once I cease using the smartphone for a certain period of time"). The compulsivity factor also implicitly indicates the state that despite the negative consequences of using smartphones, individuals could not stop them- 
selves from using smartphones (e.g., "Q5: I feel very vigorous upon the smartphone use regardless of the fatigue experiencing"). "Daily life interference" (items 6 - 8, 12, 13, 15,17 , and 18) would identify the greatest percentage of variance and inconsistency among individuals and it was one of the main factors for defining the concept of addiction to smartphones. Some items of this factor addressed the issue of interference of the smartphone use with other routine activities (e.g., "Q18: My recreational activities are reduced due to smartphone use") and some other items dealt with interpersonal problems that originated from using smartphones (e.g., "Q12: I find myself indulged on the smartphone at the cost of hanging out with friends). "Craving” (items 11, 14, 16, 19, and 21) assessed the level of one's helplessness to resist against the longing and desire for continuing the use of smartphones (e.g., "Q11: I fail to control the impulse to use the smartphone”, "Q19: I feel the urge to use my smartphone again right after I stopped using it". It also described the situations in which the individuals tend to refrain from withdrawing their addiction to smartphones (e.g., "Q14: The idea of using the smartphone comes as the first thought on my mind when waking up every morning"). Finally, the last factor namely "Sleep Interference" (items 8, 22, and 24) consisted of items focusing on the relationship between using smartphones and reduced sleep time (e.g., "Q8: I have slept less than four hours more than once due to using the smartphone") and also items that focused on sleep disorders due to smartphone use (e.g., "Q23: I make it a habit to use the smartphone despite that my sleep quality and total sleep time have decreased").

\subsection{SPAI Convergent Validity}

To test convergent validity, the correlation of SPAI scores including the SPAI total score and its five subscales' scores with the IAT total score was measured. The SPAI total score showed a significant positive correlation with the IAT total score $(r=0.73, \mathrm{P}<0.000)$. A significant correlation was found between the SPAI subscales' scores and the IAT total score, with correlation coefficients ranging from 0.647 for "craving" to 0.483 for "sleep interference" (P < $0.000)$. Table 1 presents the convergent validity results.

\subsection{Correlations Among SPAI Factors}

Internal consistency reliability (Cronbach's alpha) was performed for the revised model of the SPAI. For the SPAI total score, the Cronbach's alpha coefficient for internal consistency was excellent ( $\alpha=0.92$ ). Cronbach's alpha values for the five factors were in the range of 0.53 to 0.84 (time spent $=0.80 ;$ compulsivity $=0.65$; daily life interference
$=0.84 ;$ craving $=0.72 ;$ sleep interference $=0.53)$. Table 2 shows the correlations between the five factors.

Table 3 presents the mean and standard deviation of scores for each item of the SPAI.

\section{Discussion}

Larger proportions of the youth population in Iran are increasingly using smartphones (2). Smartphone use has definite benefits for everyday life. The smartphone technology not only has been adopted by health professionals to facilitate high-quality care $(29,30)$, but also has shown promising results to improve the self-management of chronic conditions (31-33) including substance use disorders (SUDs) $(34,35)$ through smartphone applications.

To the best of our knowledge, this is the first study that investigated the psychometric properties of the SPAI and examined its involved factors using AMOS statistical software. The results of the study showed that the Persian version of the SPAI had good internal consistency and convergent validity as a self-administrated tool for the assessment of Iranian university students. In this study, we used a confirmatory factor analysis that worked well with the five-factor solution originating from the Italian version of the SPAI (22). The authors of the Italian version of the SPAI reviewed the literature and showed that their proposed five factors were consistent with previous studies of smartphone addiction questionnaires (22). A recent study monitored the time spent on smartphones using a mobile application and showed the self-reported time of smartphones use was significantly lower than the real time of use (9).

A recent review of animal and human neuroimaging studies on the neurobiology of addiction proposed three phases of addictive behaviors toward substances including "binge and intoxication", "withdrawal and negative affect" and "preoccupation and anticipation" (35). It has been suggested that the three stages of substance addiction are also applicable to describe behavioral addiction (36) including gambling and Internet addiction disorder (37). There are similarities between "times spent," "compulsivity," and "craving" subscales of the SPAI and "binge and intoxication," "withdrawal," and "preoccupation and anticipation" subscales of substance addiction, respectively. Our five-factor model, however, did not exactly match the addiction stages proposed in the mentioned studies. Further studies are warranted to measure smartphone addiction informed by state of the art neurobiological studies.

The SPAI scores showed significant correlations with the IAT total score. This is in line with the results of another 


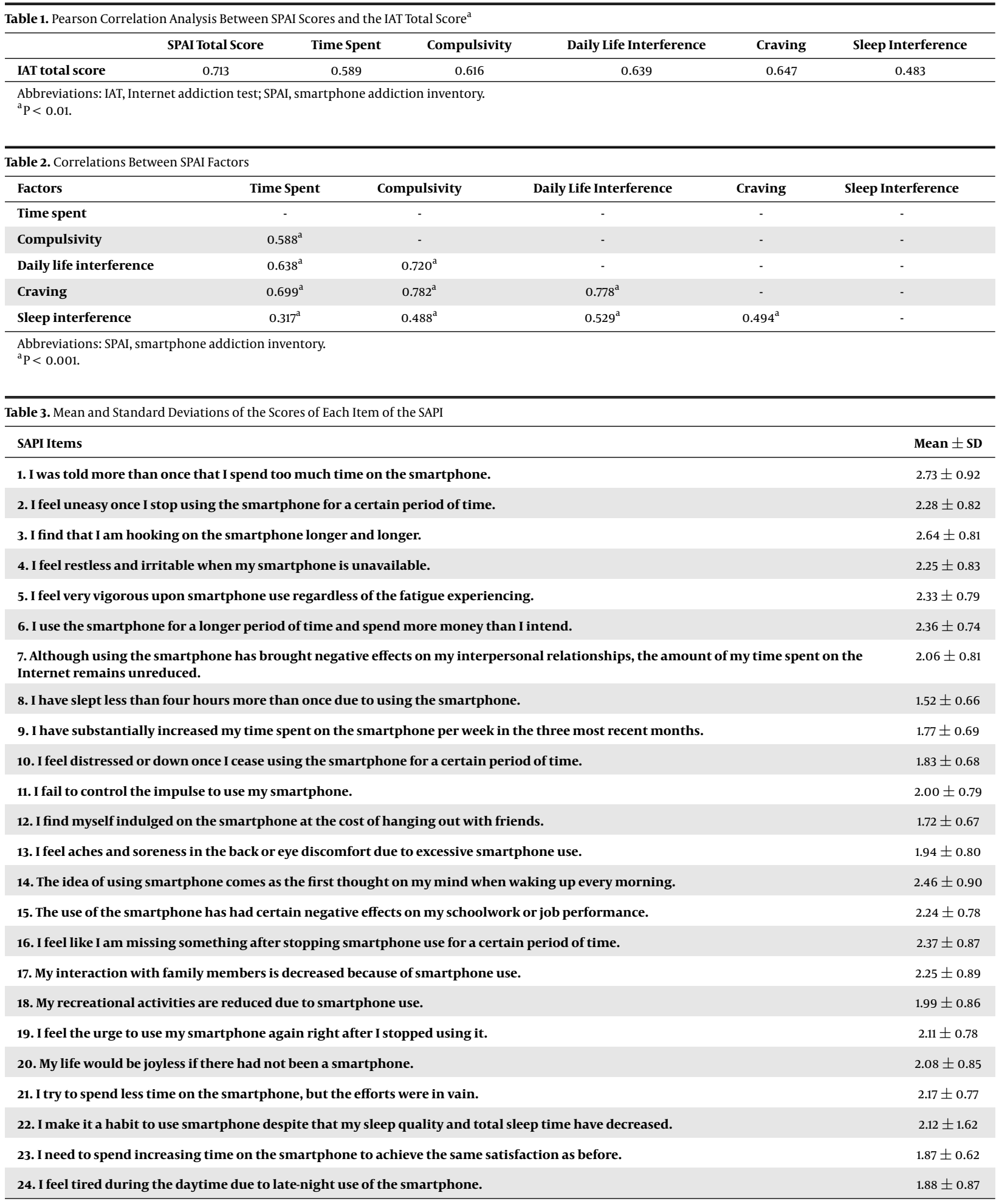

Abbreviations: SPAI, smartphone addiction inventory.

study reporting that college students with higher SPAI scores showed more preference to immediate monetary 
awards and biased toward value magnitude in intertemporal choice task, which were reported in other types of addiction (38). To better understand the nature of smartphone addiction, further research is needed on the comorbidity of smartphone addiction with other psychiatric disorders, particularly mood, anxiety, impulse control, and substance-related and addictive disorders.

\subsection{Limitations to the Study}

Study participants were all the students of Shahid Beheshti University located in the North of Tehran and most of them were female. This limits the generalizability of the results to other youth populations in Iran. More studies are needed to investigate the psychometric properties of SPAI among other Iranian populations, particularly high school students and employees at the workplace.

Recently, international studies have provided diagnostic criteria (39) and screening tools (40) for smartphone addiction. There is a need for more studies among Iranian populations to determine the cutoff scores of the SPAI. It would also be fruitful to conduct an experimental study that aims to develop a remedial program to help smartphone addicts overcome addiction, as well as to conduct research to predict factors affecting smartphone addiction among university and high school students.

\subsection{Conclusions}

The Iranian version of the SPAI showed good psychometric properties as a useful tool to measure smartphone addiction among Iranian university students. The SPAI also provided a good tool for comparing smartphone overuse among the Iranian youth and people from other countries.

\section{Footnotes}

Authors' Contribution: Saeed Imani, Alireza Noroozi, and Jaber Alizadeh Goradel conceptualized the study and drafted the manuscript. Sadegh Mousavi conceptualized the statistical procedures for the study. Alireza Noroozi and Sadegh Mousavi provided a critical revision of the manuscript. All authors read and approved the final manuscript.

Clinical Trial Registration Code: It is not to declared by the authors.

Declaration of Interest: It is not to declared by the authors.

Ethical Approval: Study protocol and assessments were approved by Ethic Committee of Shahid Beheshti University (No. 1395.781).
Funding/Support: We did not receive any fund from any organization to conduct this study.

\section{References}

1. Poushter J. Smartphone ownership and internet usage continues to climb in emerging economies. February 22, 2016. Available from http://www.pewglobal.org/2016/02/22/smartphone-ownershipand-internet-usage-continues-to-climb-in-emerging-economies/.

2. Iran ICT. Press conference of Iran ICT Minister: How many Iranians use smartphones. May 18, 2015. Available from: http://www.khabaronline. ir/detail/417657/ict/ict.

3. Grant JE, Potenza MN, Weinstein A, Gorelick DA. Introduction to behavioral addictions. Am J Drug Alcohol Abuse. 2010;36(5):233-41. doi 10.3109/00952990.2010.491884. [PubMed: 20560821]. [PubMed Central: PMC3164585].

4. Grant JE, Atmaca M, Fineberg NA, Fontenelle LF, Matsunaga $\mathrm{H}$, Janardhan Reddy YC, et al. Impulse control disorders and "behavioural addictions" in the ICD-11. World Psychiatry. 2014;13(2):1257. doi: 10.1002/wps.20115. [PubMed: 24890056]. [PubMed Central: PMC4102276].

5. American Psychiatric Association(APA).Diagnostic and statistical manual of mental disorders, 5th edition: DSM-5. 5th ed. Arlington: American Psychiatric Publishing; 2013.

6. Lin YH, Chang LR, Lee YH, Tseng HW, Kuo TB, Chen SH. Development and validation of the smartphone addiction inventory (SPAI). PLOS One. 2014;9(6). e98312. doi: 10.1371/journal.pone.0098312. [PubMed: 24896252]. [PubMed Central: PMC4045675].

7. Al-Barashdi H, Bouazza A, Jabur N. Smartphone addiction among university undergraduates: A literature review.J Sci Res Rep. 2015;4(3):21025. doi: $10.9734 /$ jsrr/2015/12245.

8. Billieux J, Maurage P, Lopez-Fernandez O, Kuss DJ, Griffiths MD. Can disordered mobile phone use be considered a behavioral addiction? An update on current evidence and a comprehensive model for future research. Curr Addict Rep. 2015;2(2):156-62. doi: 10.1007/s40429015-0054-y.

9. Lin YH, Lin YC, Lee YH, Lin PH, Lin SH, Chang LR, et al. Time distortion associated with smartphone addiction: Identifying smartphone addiction via a mobile application (App). J Psychiatr Res. 2015;65:139-45. doi: 10.1016/j.jpsychires.2015.04.003. [PubMed: 25935253].

10. Chóliz M. Mobile-phone addiction in adolescence: the test of mobile phone dependence (TMD). Prog Health Sci. 2012;2(1):33-44.

11. Billieux J, Van der Linden M, Rochat L. The role of impulsivity in actual and problematic use of the mobile phone. Appl Cogn Psychol. 2008;22(9):1195-210. doi:10.1002/acp.1429.

12. Chen L, Yan Z, Tang W, Yang F, Xie X, He J. Mobile phone addiction levels and negative emotions among Chinese young adults: The mediating role of interpersonal problems. Comput Human Behav. 2016;55:85666. doi: 10.1016/j.chb.2015.10.030.

13. Toda M, Monden K, Kubo K, Morimoto K. Mobile phone dependence and health-related lifestyle of university students. J Soc Behav Pers. 2006;34(10):1277-84. doi:10.2224/sbp.2006.34.10.1277.

14. Wang JL, Wang HZ, Gaskin J, Wang LH. The role of stress and motivation in problematic smartphone use among college students. Comput Human Behav. 2015;53:181-8. doi: 10.1016/j.chb.2015.07.005.

15. Merlo LJ, Stone AM, Bibbey A. Measuring problematic mobile phone use: Development and preliminary psychometric properties of the PUMP scale. J Addict. 2013;2013:912807. doi: 10.1155/2013/912807. [PubMed: 24826371]. [PubMed Central: PMC4008508].

16. Martinotti G, Villella C, Di Thiene D, Di Nicola M, Bria P, Conte G, et al. Problematic mobile phone use in adolescence: A cross-sectional study. J Pub Health. 2011;19(6):545-51. doi: 10.1007/s10389-011-0422-6. 
17. Roberts JA, Yaya LH, Manolis C. The invisible addiction: Cell-phone activities and addiction among male and female college students. $J$ Behav Addict. 2014;3(4):254-65. doi: 10.1556/JBA.3.2014.015. [PubMed: 25595966]. [PubMed Central: PMC4291831].

18. Bianchi A, Phillips JG. Psychological predictors of problem mobile phone use. Cyberpsychol Behav. 2005;8(1):39-51. doi 10.1089/cpb.2005.8.39. [PubMed:15738692].

19. Koo HY. [Development of a cell phone addiction scale for korean adolescents]. J Korean Acad Nurs. 2009;39(6):818-28. Korean. doi: 10.4040/jkan.2009.39.6.818. [PubMed: 20071895].

20. Kwon M, Lee JY, Won WY, Park JW, Min JA, Hahn C, et al. Development and validation of a smartphone addiction scale (SAS). PLoS One. 2013;8(2). e56936. doi: 10.1371/journal.pone.0056936. [PubMed 23468893]. [PubMed Central: PMC3584150].

21. Tossel C, Kortum P, Shepard C, Rahmati A, Zhong L. Exploring smartphone addiction: Insights from long-term telemetric behavioral measures. Int I Interact Mobile Technol (iIIM). 2015;9(2):37-43. doi: 10.3991/ijim.v9i2.4300

22. Pavia L, Cavani P, Di Blasi M, Giordano C. Smartphone addic tion inventory (SPAI): Psychometric properties and confirmatory factor analysis. Comput Human Behav. 2016;63:170-8. doi: 10.1016/j.chb.2016.05.039.

23. Terwee CB, Bot SD, de Boer MR, van der Windt DA, Knol DL, Dekker J, et al. Quality criteria were proposed for measurement properties of health status questionnaires. J Clin Epidemiol. 2007;60(1):34-42. doi: 10.1016/j.jclinepi.2006.03.012. [PubMed: 17161752]

24. Comrey LA, Lee HB. A first course in factor analysis. 2nd ed. Hillside, NJ: Lawrence Erlbaum Associates; 1992.

25. Young KS. Internet addiction: The emergence of a new clinical disorder. Cyberpsychol Behav.1998;1(3):237-44. doi: 10.1089/cpb.1998.1.237.

26. Widyanto L, McMurran M. The psychometric properties of the internet addiction test. Cyberpsychol Behav. 2004;7(4):443-50. doi: 10.1089/cpb.2004.7.443. [PubMed: 15331031].

27. Chang MK, Man Law SP. Factor structure for young's Internet addiction test: A confirmatory study. Comput Human Behav. 2008;24(6):2597-619. doi: 10.1016/j.chb.2008.03.001.

28. Mohammadsalehi N, Mohammadbeigi A, Jadidi R, Anbari Z, Ghaderi E, Akbari M. Psychometric properties of the Persian language version of yang internet addiction questionnaire: An explanatory factor analysis. Int J High Risk Behav Addict. 2015;4(3). e21560. doi: 10.5812/ijhrba.21560. [PubMed: 26495253]. [PubMed Central: PMC4609498]

29. Putzer GJ, Park Y. Are physicians likely to adopt emerging mobile technologies? Attitudes and innovation factors affecting smartphone use in the Southeastern United States. Perspect Health Inf Manag. 2012;9:1b. [PubMed: 22737094]. [PubMed Central: PMC3329206].
30. Tahamtan I, Pajouhanfar S, Sedghi S, Azad M, Roudbari M. Factors affecting smartphone adoption for accessing information in medical settings. Health Info Libr J. 2017;34(2):134-45. doi: 10.1111/hir.12174. [PubMed: 28406547].

31. Schnall R, Cho H, Mangone A, Pichon A, Jia H. Mobile health technology for improving symptom management in low income persons living with HIV. AIDS Behav. 2018;22(10):3373-83. doi: 10.1007/s10461-0172014-0. [PubMed: 29299790]. [PubMed Central: PMC6034982].

32. Brzan PP, Rotman E, Pajnkihar M, Klanjsek P. Mobile applications for control and self management of diabetes: A systematic review. J Med Syst. 2016;40(9):210. doi: 10.1007/s10916-016-0564-8. [PubMed: 27520615].

33. Plow M, Golding M. Using mhealth technology in a self-management intervention to promote physical activity among adults with chronic disabling conditions: Randomized controlled trial. JMIR Mhealth Uhealth. 2017;5(12). e185. doi:10.2196/mhealth.6394. [PubMed: 29196279]. [PubMed Central: PMC5732326].

34. Schulte M, Liang D, Wu F, Lan YC, Tsay W, Du J, et al. A Smartphone application supporting recovery from heroin addiction: Perspectives of patients and providers in China, Taiwan, and the USA.J Neuroimmune Pharmacol. 2016;11(3):511-22. doi: 10.1007/s11481-016-9653-1. [PubMed: 26846506]. [PubMed Central: PMC4974153].

35. Berman AH, Gajecki M, Fredriksson M, Sinadinovic K, Andersson C. Mobile phone apps for university students with hazardous alcohol use: Study protocol for two consecutive randomized controlled trials. JMIR Res Protoc. 2015;4(4). e139. doi: 10.2196/resprot.4894. [PubMed: 26693967]. [PubMed Central: PMC4704963].

36. Koob GF, Volkow ND. Neurobiology of addiction: A neurocircuitry analysis. Lancet Psychiatry. 2016;3(8):760-73. doi: 10.1016/S2215-0366(16)00104-8. [PubMed: 27475769]. [PubMed Central: PMC6135092].

37. Yau YH, Potenza MN. Gambling disorder and other behavioral addictions: Recognition and treatment. Harv Rev Psychiatry. 2015;23(2):13446. doi: 10.1097/HRP.0000000000000051. [PubMed: 25747926]. [PubMed Central: PMC4458066].

38. Tang Z, Zhang H, Yan A, Qu C. Time is money: The decision making of smartphone high users in gain and loss intertemporal choice. Front Psychol. 2017;8:363. doi: 10.3389/fpsyg.2017.00363. [PubMed: 28344568]. [PubMed Central: PMC5344929].

39. Lin YH, Chiang CL, Lin PH, Chang LR, Ko CH, Lee YH, et al. Proposed diagnostic criteria for smartphone addiction. PLoS One. 2016;11(11). e0163010. doi: 10.1371/journal.pone.0163010. [PubMed: 27846211]. [PubMed Central: PMC5112893].

40. Lee HK, Kim JH, Fava M, Mischoulon D, Park JH, Shim EJ, et al. Development and validation study of the smartphone overuse screening questionnaire. Psychiatry Res. 2017;257:352-7. doi: 10.1016/j.psychres.2017.07.074. [PubMed: 28800515]. 